\title{
Denosumab-Treated Giant Cell Tumor of the Bone Mimicking Low-Grade Central Osteosarcoma
}

\author{
Chang-Che Wu • Pin-Pen Hsieh \\ Department of Pathology and Laboratory Medicine, Kaohsiung Veterans General Hospital, Kaohsiung, Taiwan
}

Giant cell tumor of the bone is a benign but locally aggressive bone tumor. In recent years, denosumab, a receptor activator for nuclear factor- $\kappa \beta$ ligand (RANKL) inhibitor has emerged as a treatment alternative, especially when surgical clearance cannot be obtained or for frequent recurrence. However, treatmentrelated histologic changes have been rarely documented in the literature until recently and show substantial overlap with other primary tumors of bone such as low-grade central osteosarcoma, which can easily lead to a misdiagnosis. Here we present a typical case of denosumab-treated giant cell tumor of the bone confirmed by detection of an $\mathrm{H} 3 \mathrm{~F} 3 \mathrm{~A}$ (histone $\mathrm{H} 3$ family $3 \mathrm{~A}$ protein) mutation, which we believe is the first case report in Taiwan.

\section{CASE REPORT}

A 50-year-old female patient visited our clinic complaining of left knee pain that had been present for 4 months. The condition was aggravated during ambulation and worsened as time went by. There was no trauma history or any significant past medical history of note. She had gone to another hospital first where a left distal femur osteolytic tumor was found. Needle biopsy was performed there, and the pathology revealed giant cell tumor of the bone (Fig. 1A). She was then given subcutaneous injections of denosumab (120 mg) every 4 weeks for 3 months. Due to personal reasons, she visited our clinic thereafter. Magnetic resonance imaging showed a 4.1-cm expansile, eccentric osteolytic

\section{Corresponding Author}

Pin-Pen Hsieh, MD

Department of Pathology and Laboratory Medicine, Kaohsiung Veterans General

Hospital, 386, Ta-Chung 1st Rd., Kaohsiung 813, Taiwan

Tel: +886-7-3422121 (ext. 6306), Fax: +886-3422288, E-mail: pinpenh@gmail.com

Received: September 30, 2016 Revised: December 8, 2016

Accepted: December 21, 2016 bone tumor around the epiphysis and metaphysis of the left distal femur; the lesion manifested low T1 weighted image and T2 weighted image with strong contrast enhancement (Fig. 1B). Relatively well-defined margins with cortical thinning were noted. No conspicuous tumor regression was noted, and the lesion was still radiologically classified as grade II using the Campanacci classification. After 3 months of denosumab treatment, adequate curettage and adjuvant cryotherapy with liquid nitrogen and bone graft implantation were performed.

A brownish circumscribed tumor, $5 \mathrm{~cm}$ in size, was found during the operation, with no soft tissue or joint involvement. The curettage specimen was sent for pathologic examination. Light microscopy showed that the tumor was composed mostly of woven bone, with a small amount of aggregated mononuclear cell foci and scattered osteoclast-like giant cells $(<10 \%)$. Compared with ordinary giant cell tumors of the bone before denosumab treatment (Fig. 1A), this post-treatment specimen showed decreased cellularity, reduced number and size of osteoclast-like giant cells, and abundant new bone deposition as broad, rounded cords, or long, curvilinear arrays (Fig. 1C, D). Only minimal cytological atypia and occasional mitoses were noted. Focal necrosis was identified. Immunohistochemically, the tumor cells were negative for cyclin-dependent kinase 4 (1:200, ZETA Corporation, Arcadia, CA, USA), and murine double minute 2 (MDM2; 1:50, ZETA Corporation) (Fig. 1E). The genetic study was negative for $M D M 2$ amplification. In addition, the $H 3 F 3 A$ gene mutation was assessed with polymerase chain reaction and direct sequencing which showed hotspot mutation c.100G $>$ T (G34W) (Fig. 1F).

After the operation, the range of motion (ROM) for the left knee gradually improved from partial to full ROM at 6-month follow-up. No evidence of tumor recurrence was noted. 
This study was approved by the Institutional Review Board of Kaohsiung Veterans General Hospital (IRB No. VGHKS18CT2-01) with a waiver of informed consent.

\section{DISCUSSION}

Giant cell tumor of the bone is composed of a proliferation of mononuclear cells amongst which are scattered numerous macrophages and large osteoclast-like giant cells. It is thought that the numerous large osteoclast-like giant cells are not neoplastic but reactive in nature. ${ }^{1}$ These neoplastic mononuclear cells exhibit mutations to the $H 3 F 3 A$ gene, ${ }^{2}$ and express high levels of RANKL, ${ }^{3}$ which binds to RANK, a receptor expressed on the surface of multinucleated osteoclast-like giant cells and their precursors. This results in the activation and proliferation of these cells, leading to bone resorption. ${ }^{4}$ Denosumab, a RANKL inhibitor, has been shown to retard or arrest tumor growth. ${ }^{5}$ Denosumabtreated tumors can be devoid of giant and mononuclear cells and are composed of abundant woven bone and fibrous tissue.

Wojcik et al. ${ }^{6}$ reported nine cases and demonstrated that all treated giant cell tumors of the bone showed marked giant cell depletion. A gradual decrease in stromal cellularity and increased
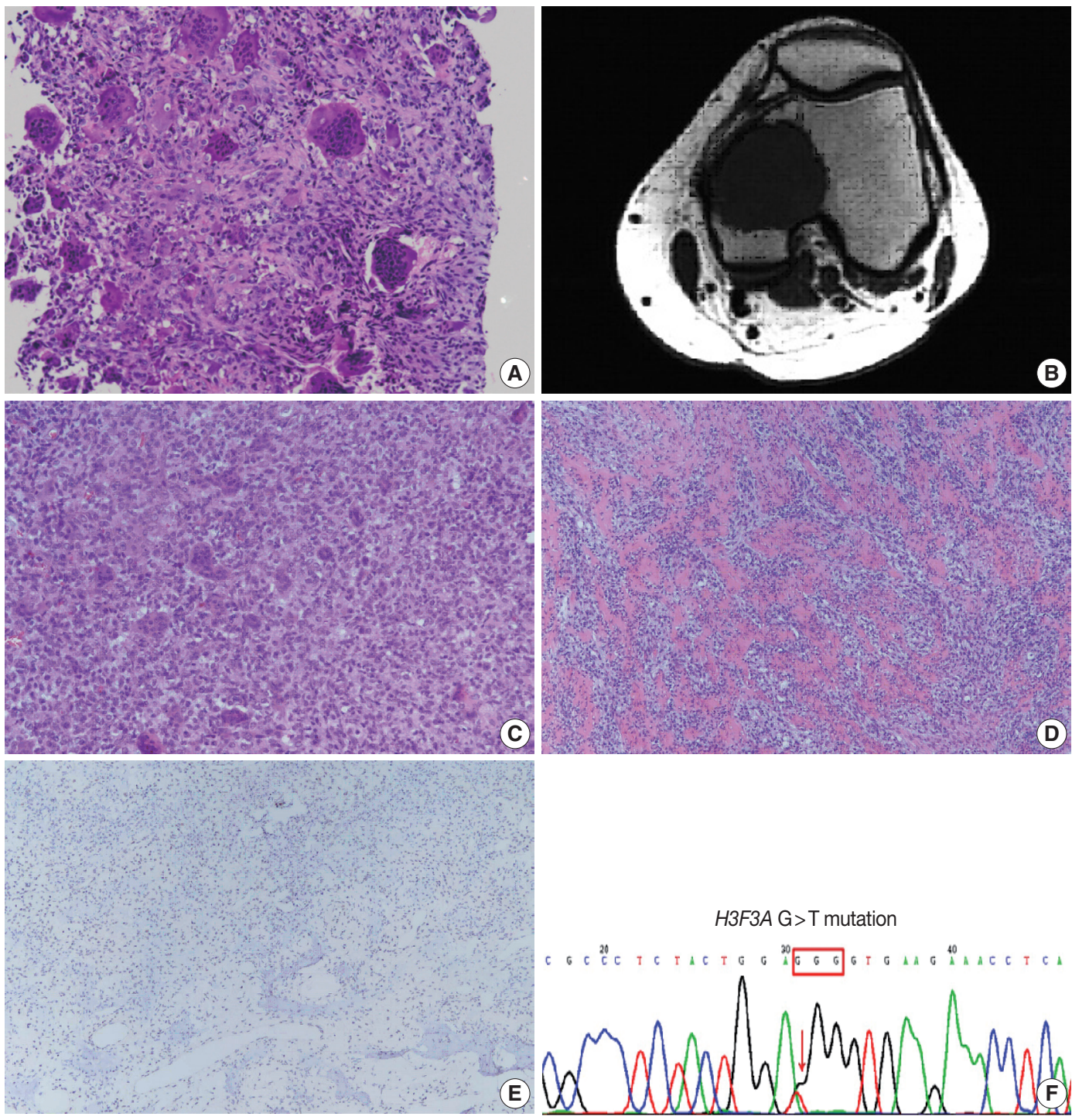

Fig. 1. (A) The typical picture of giant cell tumor before treatment shows "giant" osteoclasts with $>20$ and sometimes $>50$ nuclei. (B) Magnetic resonance imaging reveals an expansile, eccentric osteolytic bone tumor around epiphysis and metaphysis of left distal femur. (C, D) Compared with ordinary giant cell tumor, this case exhibits reduced number and size of osteoclast-like giant cells and also abundant new bone deposition, mimicking low-grade central osteosarcoma. (E) The immunohistochemical result shows negative for MDM2. (F) H3F3A hotspot mutation (G34W) is detected. 
new bone formation appeared to correlate with treatment. Denosumab shifts the balance of physiological bone formation away from RANK-mediated osteoclastic bone resorption toward osteoprotegrin-induced bone formation. However, it seems that this shift does not reflect terminal differentiation, as the morphology reverts to classic giant cell tumor histopathology upon denosumab therapy cessation.

The combination of relatively bland-looking tumor cells with minimal cytological atypia, as well as abundant new bone formation in curvilinear arrays within a fibrous background, produced a resemblance to low-grade central osteosarcoma. ${ }^{6}$ In addition, benign multinucleated giant cells have been reported in up to $36 \%$ of low-grade central osteosarcomas, making the distinction more challenging. Recently, Rekhi et al. ${ }^{8}$ also reported that after denosumab treatment, giant cell tumors of the bone can appear as low-grade osteosarcomas on histopathologic examination, but lack the clinical behavior of an osteosarcoma.

In terms of differential diagnoses, giant cell-rich conventional osteosarcoma and malignancy in giant cell tumor of the bone are characterized by more severe cytological atypia and more prominent mitotic activity, which were not seen in this case. Low-grade central osteosarcoma was also excluded due to the lack of an infiltrative growth pattern and negative $M D M 2$ amplification. Finally, we performed a genetic test and an H3F3A hotspot mutation $(\mathrm{G} 34 \mathrm{~W})$ was detected. This finding supported the diagnosis of giant cell tumor of the bone.

In summary, we present a case of giant cell tumor of the bone with typical morphologic changes after denosumab treatment, including giant cell depletion and abundant new bone formation. Detection of an H3F3A hotspot mutation (G34W) also confirmed the diagnosis. This is the first case report in Taiwan. Because denosumab-treated tumors bear little resemblance to their pretreatment counterparts and have substantial histologic overlap with other primary tumors of the bone, it is important for pathologists to understand the differences between treatmentrelated histologic changes and morphologic mimics. Due to the increasing use of denosumab in recent years, it is crucial to pay careful attention to the history of denosumab administration to avoid a misdiagnosis.

\section{Conflicts of Interest}

No potential conflict of interest relevant to this article was reported.

\section{Acknowledgments}

We thank Dr. Hsuan-Ying Huang, the director of Department of Pathology, Kaohsiung Chang Gung Memorial Hospital, who helped us perform the molecular study of MDM2 amplification and $H 3 F 3 A$ mutation.

\section{REFERENCES}

1. Fletcher CD, Bridge JA, Hogendoorn PC, Mertens F. WHO classification of tumours of soft tissue and bone. 4 th ed. Lyon: IARC Press, 2013; 321-4.

2. Behjati S, Tarpey PS, Presneau N, et al. Distinct H3F3A and H3F3B driver mutations define chondroblastoma and giant cell tumor of bone. Nat Genet 2013; 45: 1479-82.

3. Zheng MH, Robbins P, Xu J, Huang L, Wood DJ, Papadimitriou JM. The histogenesis of giant cell tumour of bone: a model of interaction between neoplastic cells and osteoclasts. Histol Histopathol 2001; 16: 297-307.

4. Cowan RW, Singh G. Giant cell tumor of bone: a basic science perspective. Bone 2013; 52: 238-46.

5. Thomas D, Henshaw R, Skubitz K, et al. Denosumab in patients with giant-cell tumour of bone: an open-label, phase 2 study. Lancet Oncol 2010; 11: 275-80.

6. Wojcik J, Rosenberg AE, Bredella MA, et al. Denosumab-treated giant cell tumor of bone exhibits morphologic overlap with malignant giant cell tumor of bone. Am J Surg Pathol 2016; 40: 72-80.

7. Kurt AM, Unni KK, McLeod RA, Pritchard DJ. Low-grade intraosseous osteosarcoma. Cancer 1990; 65: 1418-28.

8. Rekhi B, Verma V, Gulia A, et al. Clinicopathological features of a series of 27 cases of post-denosumab treated giant cell tumors of bones: a single institutional experience at a tertiary cancer referral centre, India. Pathol Oncol Res 2017; 23: 157-64. 